\title{
Mechanical Properties of Glass Fiber Composites Reinforced by Textile Fabric
}

\author{
Arturs Macanovskis, Andrejs Krasnikovs, Olga Kononova, Galina Harjkova, \\ Vladislav Yevstignejevs \\ Riga Technical University, Institute of Mechanics, Riga, Latvia
}

\begin{abstract}
Interest to structural application of textile reinforced polymer matrix composite materials (CM) is growing during last years. In different branches of machine building, aerospace, automotive and others industries we can find structural elements preferably be produced using such reinforcement. At the same time, such materials are exhibiting elastic and strength properties scatter. Present work is devoted to structural modeling of the composite material with textile (knitted) reinforcement having the goal to predict such materials strength and behavior under applied mechanical loads. In the framework of the present investigation, we observe yarn penetrated by a resin in a composite as a reinforcing "macro" fiber. Such "macro" fiber mechanical properties were measured experimentally, for this purpose was produced and was tested by tension until fracture fiber samples, having different length. Then was elaborated and was realized structural strength probabilistic model. In the textile geometry, was picked out repeating structural element - polymer matrix volume with two curved "macro" fiber's chunks inside it. Complete composite material volume is possible to represent as a set of repeating structural elements. External loads application leads to disperse structural elements failure. Neighboring to ruptured elements are overloaded leading to higher probability to fail for them. Using FEM was modeled stress state in "macro" fibers inside CM. Then, was numerically obtained stress distribution in composite material, having different number of broken loops. Probabilities of different numbers of failed elements were calculated. Strength probability function, based on Weibull approach was obtained. CM samples were tested under tension and obtained results were compared with numerical modeling as well as were analyzed.
\end{abstract}

Keywords: Elastic properties, glass fiber, polymer matrix, yarn.

\section{INTRODUCTION}

Novel composite materials (CM) investigation and incorporation in different structural automotive, marine and aerospace applications is important nowadays. Some of such materials have nontraditional reinforcement. Polymer matrix composites, reinforced by textile fabric in many situations have simple fabrication technology and are exhibiting attractive mechanical properties, such as high wear and impact resistance, as well as high energy absorption. Woven fabric has threads running horizontally and vertically, in the case of knitted fabric, strands are forming loops. Knitted fabric has high shape forming ability, which allows it to be applied in a complex shape mould without forming folds. Such property is important in fabrication of composite material made sophisticated form structural elements. Elastic properties of a composite reinforced by knitted fabric are dependent on mechanical properties of the matrix and mechanical properties of the fibers as well as thread thickness, its degree of twist, geometrical size and form of the loops in the fabric and were numerically and experimentally investigated in [1-6]. At the same time strength properties and damage accumulation in such materials during loading are investigated poor. Damage accumulation in mechanically loaded $\mathrm{CM}$, reinforced by knitted fabric is stochastic process of single disperse yarn loops rupture, ruptured loops formation in clusters and clusters growth till final material rupture within structural element. If we want to model such process numerically we can observe yarn filled by polymer matrix as a "macro" fiber having elastic properties corresponding to curved unidirectional composite material and strength properties which can be obtained experimentally performing such unidirectional composite material testing. After that stresses in curved "macro" fiber loops can be calculated numerically using FEM approach. More overloaded crossections of "macro" fiber loops in the material will fail. Performing stochastic procedure is possible to evaluate this process from the beginning till the final $\mathrm{CM}$ rupture. In such case studied materials have properties necessary for design and manufacturing. 


\section{UNIDIRECTIONAL MATERIAL EXPERIMENTAL INVESTIGATION}

We observe yarn filled by polymer matrix as a "macro" fiber having elastic properties corresponding to curved unidirectional composite material. Strength properties of such CM will be evaluated stochastically using Weibull probabilistic distribution (1). Weibull probabilistic distribution

$$
\begin{aligned}
& F(\sigma)=1-e^{\left(-\left(\frac{\sigma}{\eta}\right)^{\beta}\right)} \\
& \text { where: } \eta=\left(\frac{L_{0}}{L}\right)^{\frac{1}{\beta}} * \sigma_{0}
\end{aligned}
$$

(1) will describe "macro" fiber probability to fail dependent on applied to the composite "macro" fiber stretching stress $\sigma$ and its length $L . \sigma_{0}, \beta, L_{0}$ are numerical constants. In the framework of our investigation, we are accepting that curved "macro" fiber in the composite can fail only by its rupture

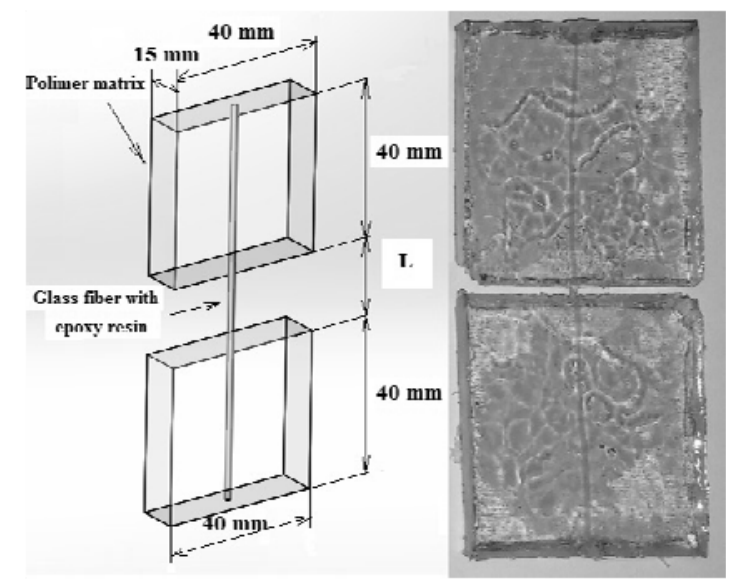

Fig. 1. Sample M2, where $\mathrm{L}-$ the length between the tabs in $\mathrm{mm}$

under acting in its orthogonal to longitudinal fiber direction tensile stress. Traditional way of parameters $\eta$ and $\beta$ experimental evaluation is performing experimental tests with fibers having different length. For this purpose composite "macro" fiber samples having different span length were experimentally fabricated. For getting samples - were conducted following steps: a) into epoxy resin was poured catalyzer for gradual solidification; b) straight glass fiber yarns were easily stretched and were penetrated by epoxy resin. After solidification "macro" fibers were cutted in pieces for samples fabrication. Each "macro" fiber piece was precisely positioned going throw two empty boxes with the size 40 X40X15 mm as is shown in Fig. 1. Boxes were filled by a resin. After 2 days epoxy resin was hardened and samples were demoulded and tested by tension till rupture. Corresponding following samples were fabricated:

1) M2 - samples with a "macro" fiber length between tabs $\mathrm{L}=2 \mathrm{~mm}$;
2) M6 - samples with a "macro" fiber length between tabs $\mathrm{L}=6 \mathrm{~mm}$;

3) M10 - samples with a "macro" fiber length between tabs $\mathrm{L}=10 \mathrm{~mm}$;

4) M16 - samples with a "macro" fiber length between tabs $\mathrm{L}=16 \mathrm{~mm}$;

5) M26 - samples with a "macro" fiber length between tabs $\mathrm{L}=26 \mathrm{~mm}$;

6) M100 - samples with a "macro" fiber length between tabs $\mathrm{L}=100 \mathrm{~mm}$;

7) M800 - samples with a "macro" fiber length between tabs $\mathrm{L}=800 \mathrm{~mm}$;

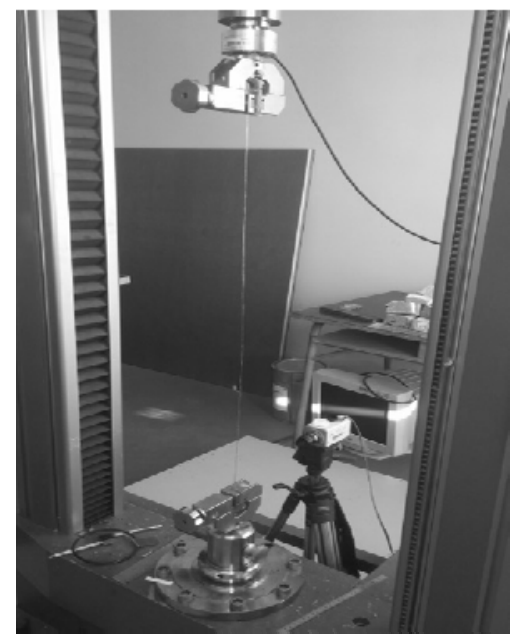

Fig. 2. The sample M800 is testing

Were produced five samples having each particular L length value between tabs. Samples were placed in a tensile testing machine grips (Fig. 2) and subjected to tension.

\section{III “MACRO” FIBER ELASTIC PROPERTIES}

"Macro" fiber consists of glass fiber yarn penetrated by epoxy resin. At the beginning, dry glass fibers yarn without epoxy resin was mechanically investigated. Yarn was tested by tension for finding Young's modulus. The diameter of the glass fiber yarn was calculated by the following (3) [7]:

$$
\mathrm{D}=\frac{\sqrt{\frac{4}{\pi * \rho * k}}}{\sqrt{1000}} * \sqrt{T e x}
$$

where: $\mathrm{D}$ - diameter of the yarn in $\mathrm{mm}$;

Tex - is a unit of measuring the linear mass density of fibers, $300 \mathrm{~g} / \mathrm{km}$;

$\rho$-the density of the glass fiber, $2.54 \mathrm{~g} / \mathrm{cm} 3$

$\mathrm{k}$ - numerical coefficient, which describes how many fibers are in the yarn volume.

Experimental curve averaged over data obtained for five samples is shown in Fig. 3. Young's modulus for a glass fiber yarn without epoxy resin was obtained 
$\mathrm{E}=71.9 \mathrm{GPa}$. Then all prepared "macro" fiber samples having different

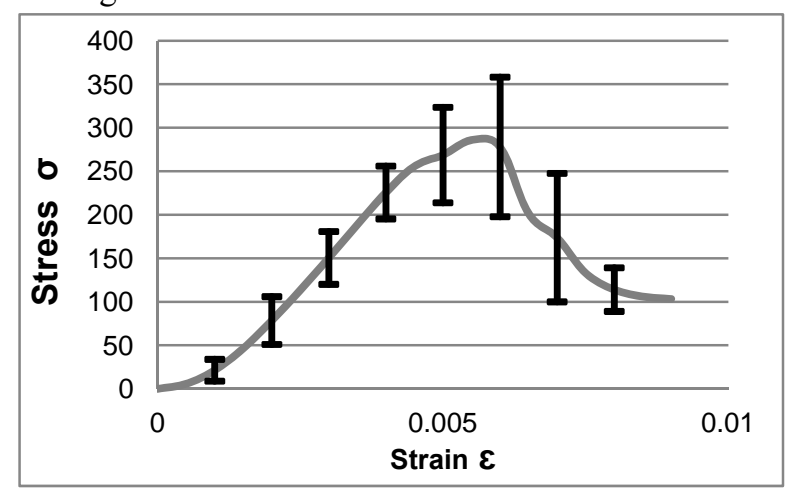

Fig. 3. Stress (MPa ) -strain (averaged over five samples) curve for glass fiber yarn not contains epoxy resin

length were tested. During samples testing was observed following:

a) for some part of M2 samples fiber break was happened partially inside the clamps. Broken crossection was inside polymer tabs;

б) for all samples M6, M10, M16, М26 иМ100 "macro" fiber broken crossection was located in the middle part of the sample away from the polymer tabs (Fig. 4);

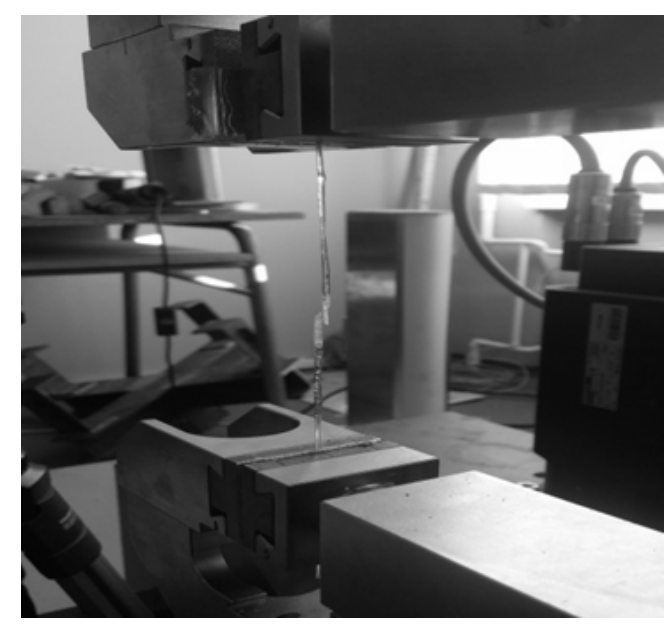

Fig. 4. Broken sample M100

c) for samples M800 "macro" fiber broken crossection was located in the middle part of the sample. First break was initiated elastic wave which running from broken crossection along the "macro" fiber in both directions was initiated secondary breaks, leading to sample separation in few pieces.

After testing for samples were received following graphs:

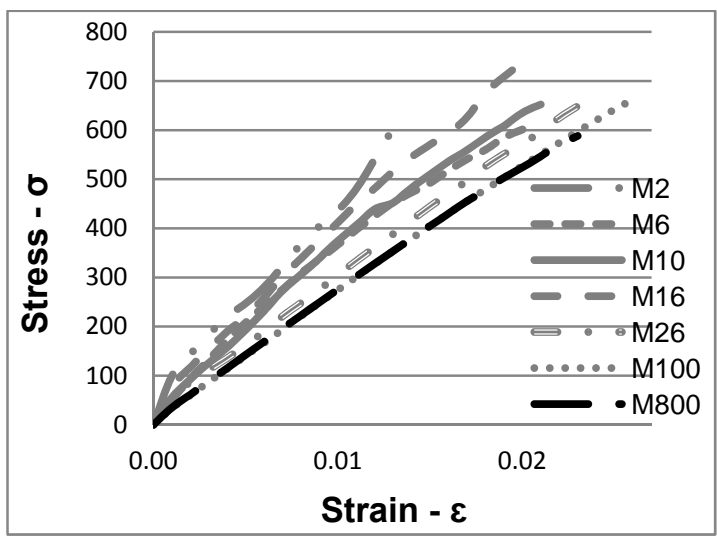

Fig. 5. Stress $(\mathrm{MPa})$ - strain (each averaged over five samples) curves for all tested samples

From curves in the Fig. 5 were obtained Young's modulus of the "macro" fiber $\mathrm{E}_{\text {average }}=32.4 \mathrm{GPa}-$ the average value over all experimentally obtained data. The average volumetric glass fibers content in our prepared samples was evaluated from the (4) and was $V_{f}=0.42$.

$$
V_{f}=\frac{E_{m}-E_{\text {average }}}{E_{m}-E_{f}}
$$

where: $\mathrm{E}_{m}=4 \mathrm{GP}-$ Young's modulus of the epoxy matrix, $\mathrm{E}_{f}=71.9 \mathrm{GPa}$ - the average value of the Young's modulus of the glass fiber without epoxy resin, which was obtained in experiments stretching yarns without resin.

\section{IV “MACRO” FIBER STOCHASTIC PROPERTIES}

All length samples were tested till rupture. Strength properties dependence on samples length is shown in Fig. 6. From Fig. 6 were obtained following approximation function $\ln (\mathrm{L})=-8.0033 * \ln (\sigma)+55.366$ and values $\beta=8.1443$ and $A=56.709$ in Weibull probabilistic function (1) and (2) describing glass fiber yarn impregnated by epoxy matrix strength distribution.

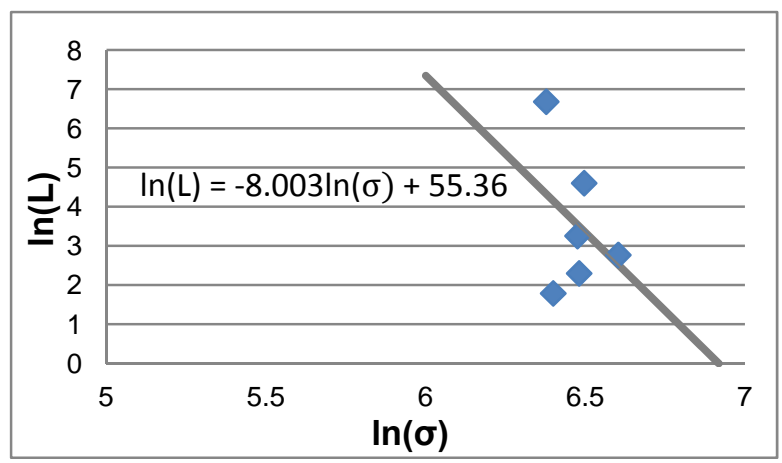

Fig. 6. Linear approximation of the samples strength dependence on length for "macro" fibers, were L is in $\mathrm{mm}$ and $\sigma$ in $\mathrm{MPa}$ 


\section{$\mathrm{V}$ DAMAGE ACCUMULATION IN THE COMPOSITE MATERIAL REINFORCED BY KNITTED FABRIC AND IT STRENGTH}

Existing failure models are mostly based on the use of different phenomenological criteria [5,6,8]. Polymer matrix composite material reinforced by knitted fabric is forming repeatable structure of elements shown in Fig. 7. A repeating structural element - volume can be subtracted from the structure. Translation of the volume in four directions gives us composite plate. Such subtracted volume of looped fiber with surrounding matrix is shown in Fig. 8. In the case of such material loading, all translated repeatable volumes are loaded in the same way. The finite element method was used studying sequential failure of loops in a plate of composite material reinforced with knitted fabric. Loading of material will lead to distributed in CM volume "macro" fiber breaks within the loops. We suppose then if failure will happen it will happen in more heavily loaded crossection of the loop. For this purpose more stretched crossection within structural element was recognized (Fig. 9). Then according to applied external loads level, probabilities to find such single breaks dispersed in material were calculated according to (1), using obtained experimentally numerical constants. The broken crossection within the loop is leading to overstress formation on crossections within closest neighboring fibers (in neighboring repeating volumes). More heavily loaded crossection of the fiber within neighboring to ruptured loop was recognized and probability to failure under overloads was obtained. In such way, probabilities to find in loaded CM plate single ruptured "macro" fibers crossections, two neighboring broken crossections in two neighboring "macro" fibers, three broken crossections in three neighboring "macro" fibers were calculated and so on.

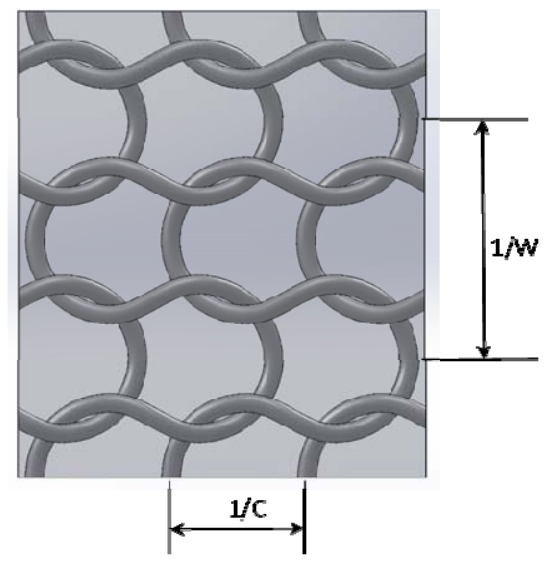

Fig. 7. Polymer matrix composite plate reinforced by glass fiber knitted fabric. Glass fiber yarn impregnated by epoxy matrix is observed as "macro" fiber
Thin knitted reinforcement in the composite is shown in Fig. 7. This structure is loaded with a tensile force in the horizontal direction. Investigated composite material plate sizes was $12 \times 14 \times 2.43 \mathrm{~mm}$ (width $\times$ length $\times$ thickness). Knitted geometric parameters were: number of loop columns per $\mathrm{cm}$, $\mathrm{W}=1.43 \mathrm{loop} / \mathrm{cm}$, the number of rows per $\mathrm{cm}, \mathrm{C}=2.5$ loop $/ \mathrm{cm}$. In order to simulate the thin knitted fabric geometry was used Leif-Glaskin model [1-4].

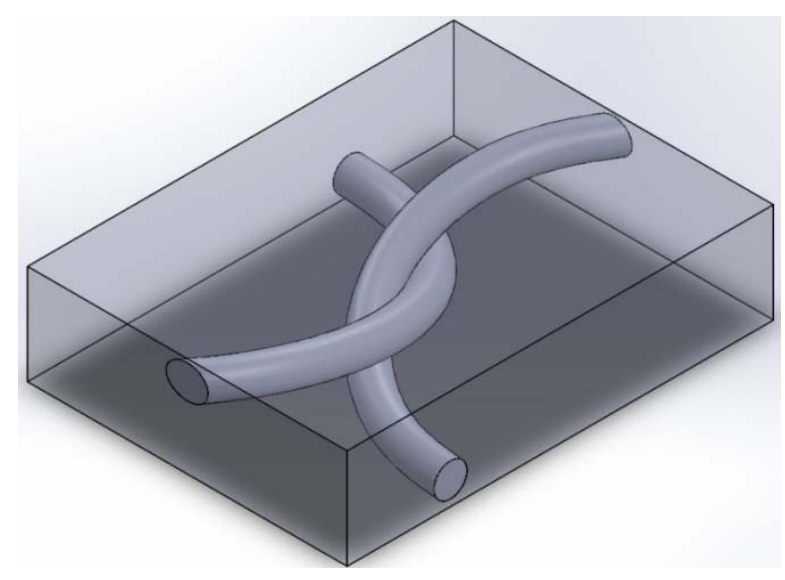

Fig. 8. Repeating structural element of the composite material [1-3]

Applied tensile stress in horizontal direction causes uneven tensile stress distribution along "macro" fiber orthogonal crossections.

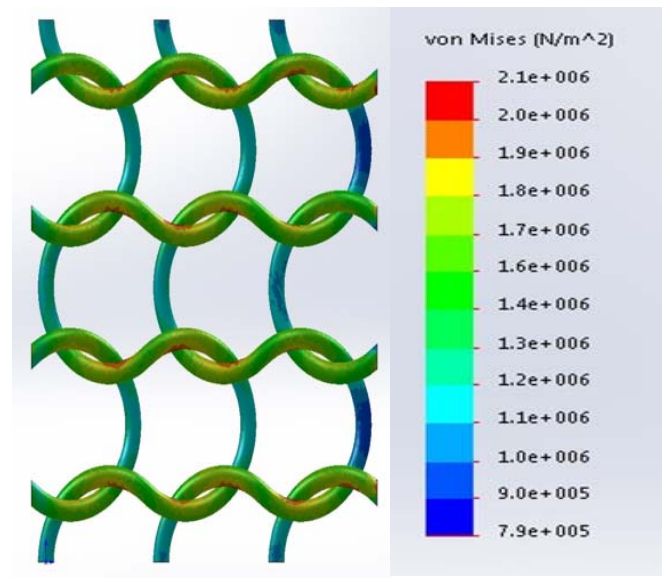

Fig. 9. Equivalent Mizes stress distribution in orthogonal crossections of „macro" fibers in a case of CM stretching in horizontal direction by stress equal to $1 \mathrm{MPa}$ (FEM solution)

Was supposed full contact between „macro" fibers and matrix. Was used FEM mesh, dependent on the curvature of the fiber. Finite element maximal size was $0.1675 \mathrm{~mm}$. Were calculated stresses in situations of one broken crossection, two broken crossection, three broken crossection and so on till 12. Stress distribution along unbroken fiber within the repeating element is show in Fig. 9 and Fig.10. The averaged (across crossection of the "macro" fiber) overload 
coefficients in more dangerously loaded neighboring fibers are shown in the table 1.

TABLE I

\begin{tabular}{|c|c|}
\hline $\begin{array}{c}\text { Number of ruptured } \\
\text { crossections }\end{array}$ & $\begin{array}{c}\text { Normal stress in more heavily } \\
\text { loaded neighboring } \\
\text { crossection, MPa }\end{array}$ \\
\hline $\begin{array}{c}\text { Material without broken } \\
\text { crossections }\end{array}$ & 1.7640 \\
\hline 1 broken crossection & 1.7660 \\
\hline 2 broken crossections & 1.7680 \\
\hline 3 broken crossections & 1.7700 \\
\hline 4 broken crossections & 1.7710 \\
\hline 5 broken crossections & 1.7714 \\
\hline 6 broken crossections & 1.7732 \\
\hline 7 broken crossections & 1.7750 \\
\hline 8 broken crossections & 1.7768 \\
\hline
\end{tabular}

Simulation of damage accumulation in the composite material having knitted reinforcement based on the assumption that it is a complex stochastic process, which begins with the single ruptured yarns accumulation in the material and is continuing to such single ruptures growth by other yarns failure on the border of the cluster of ruptured commonly located yarns. This process begins relatively slowly and turns to catastrophic development when the cluster growth. Model is not considering interaction between two adjacent clusters.

Let introduce the random value $I(\sigma): P\{I(\sigma) \geq$ $i, \sigma>0$, it is the size of neighboring broken fibers crossections. Cluster is reaching size of at least $i$ adjacent broken yarns when the external tensile load is reaching value $\sigma$. In this case probability to find inside material clusters having size at least one broken "macro" fiber is equal to $P(i \geq 1)=W(\sigma)$. The probability of finding clusters, consisting of at least two broken adjacent „macro" fibers is equal to $P(i \geq 2)=P(i \geq 1) W\left(\sigma_{1}\right)$, the probability of finding clusters, consisting of at least three breaking adjacent „macro" fibers is $P(i \geq 3)=P(i \geq 2)\left(1-\left(1-W\left(\sigma_{2}\right)\right)^{2}\right)$, the probability of finding clusters, consisting of at least four breaking adjacent "macro" fibers is $P(i \geq 4)=P(i \geq 3) W\left(\sigma_{3}\right)$.

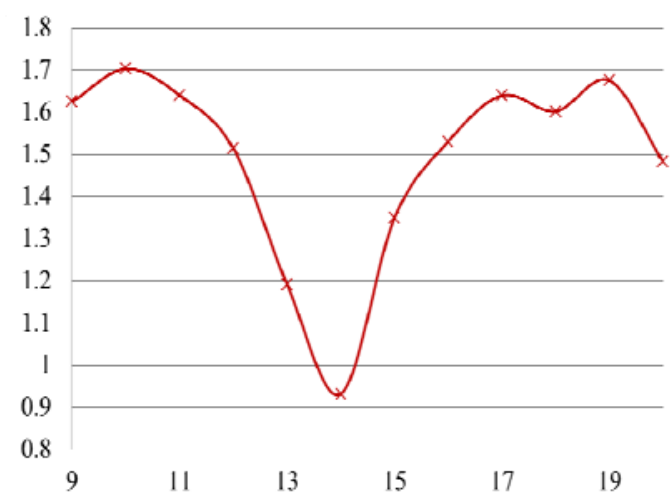

Fig. 10. The averaged overload coefficient distribution for (across crossection of the "macro" fiber) tensile stress acting along the fiber within one repeating structural element
This process can be continued and probability of finding clusters, consisting of at least $n$ breaking adjacent ,macro" fibers can be calculated as $P(i \geq n)=P(i \geq n-1) W\left(\sigma_{n-1}\right)$.

Above mentioned probabilistic model was realized and probabilities to find different size clusters of adjacently located broken fibers were obtained (Fig. 11). When size of the cluster is growing, probabilistic curves are linking forming one envelop curve, which is CM plate failure curve (probability to find in the plate cluster consisting of $n \rightarrow \infty$ broken crossections).

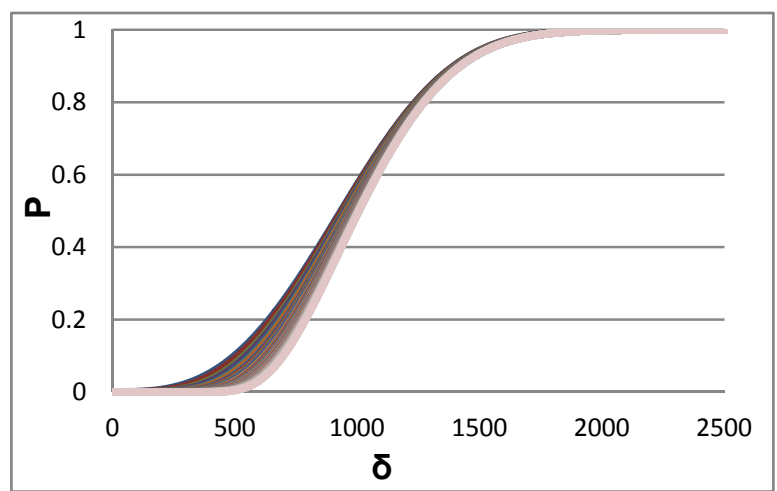

Fig. 11. Probabilities to obtained different size clusters consisting of broken adjacent "macro" fibers in loaded by tension CM plate

\section{CONCLUSION}

In the present work detailed failure process investigation in mechanically loaded $\mathrm{CM}$ plates reinforced by knitted fabric was realized. For this purpose experimentally were obtained micro composite material - single glass fiber yarn in epoxy resin, elastic and strength properties. Probabilistic numerical model was elaborated for structural damage accumulation evaluation. Model was realized and probability of $\mathrm{CM}$ plate reinforced by knitted glass fiber yarn was obtained.

\section{REFERENCES}

[1] Ramakrishna, S and Hull, D., Tensile Behavior of Knitted Carbon-Fibre-Fabric/Epoxy Laminates, Composites Science and Technology, Vol. 50, 1994, pp.237-247.

[2] A.Krasnikovs, O.Kononova, G.Harjkova, V.Zaharevskis and A.Galushchak „Mechanical Properties Characterization by Inverse Technique for Composite Reinforced by Knitted Fabric", CD-Proceedings of ECCM15 - 15th European Conference on CompositeMaterials, Venice, Italy, 24-28 June 2012,8 p.

[3] Kononova, O., Krasņikovs, A., Harjkova, G., Zal̦eskis, J., Mačanovskis, E. Characterization of Mechanical Properties by Inverse Technique for Composite Reinforced by Knitted Fabric. Part 1. Material Modeling and Direct Experimental Evaluation of Mechanical Properties. Journal of Vibroengineering, 2012, Vol.14, Iss.2, pp.681-690.

[4] Kononova, O., Krasnikovs, A., Dzelzītis, K., Harjkova, G., Vagele, A., Eiduks, M. Modelling and Experimental Verification of Mechanical Properties of Cotton Knitted Fabric Composites. Estonian Journal of Engineering, 2011, Vol.17, Iss.1, pp.39-50.

[5] Ramakrishna, S., Cuong, N.K. and Hamada, H., Tensile Properties of Plain Weft Knitted Glass fiber Fabric Reinforced 
Epoxy Composites, J. Reinf. Plast. \& Comp. Vol. 16, No 10, 1997, pp. 946-966.

[6] Luo, Y.; Verpoest, I. Biaxial tension and ultimate deformation of knitted fabric reinforcements, Compos. Part A: Appl. Sci. Manuf. 2002, 33, 197-203.
[7] Matusiak $M$ and Sikorski K. Influence of the structure of woven fabrics on their thermal insulation properties. Fibres Text East Eur 2011; 19: 46-53.

[8] Ramakrishna S., Fujita A., Cuong N.K., Hamada H. Tensile failure mechanisms of knitted glass fibre fabric reinforced epoxy composites. (Proc. Conf.) Fourth Japan International SAMPE Symposium, 25-28 September 1995. pp. 661-666. 